\title{
“CAN MIGRANTS ACT?”. PRESENZA, ORGANIZZAZIONE, VISIBILITÀ IN UN ORIZZONTE PRECARIO
}

\author{
"Can migrants act?". Presence, Organization, Visiblity \\ in a Precarious Scape
}

Filippo Furri ${ }^{1}$

\begin{abstract}
Riassunto. L'articolo prende spunto dalla vicenda della jungle di Calais per interrogare i margini e le condizioni di un agire migrante nell'orizzonte europeo. Riferendosi al celebre articolo di Spivak "Can Subaltern Speak", il contributo vuole considerare il processo di neutralizzazione (giuridica e esistenziale) che investe il migrante nelle sue varie fasi e situazioni di accesso alla società di "accoglienza", e il suo impatto sulla soggettività migrante, prima di illustrare alcune declinazioni di un agency che cerca di smarcarsi rispetto al dispositivo in essere.
\end{abstract}

Parole chiave: presenza/crisi delle presenza, agency, Calais, neutralizzazione, organizzazione.

\begin{abstract}
Starting from Calais jungle's events, this paper investigates the margins and the conditions of a migrant agency into the European horizon. Referring to the famous Spivak's paper "Can Subaltern Speak", this contribution means to consider the neutralization process (in a juridical and existantial pov), investing the migrant in different phases and steps in order to yield to the "host" society, and the impact that all of this has on the migrant's subjectivity. In its final part, the paper describes some examples used by an agency which tries to avoid this capture and neutralization dispositive.
\end{abstract}

Keywords: presence/presence crisis, agency, Calais, neutarlization, organization.

\section{La jungle di Calais}

Nel corso di un intervento presso una scuola per operatori sociali di Lille (IRTS) per presentare una ricerca sulla nozione di città-rifugio come modello

\footnotetext{
1 Phd Candidat, Département d'anthropologie Université de Montreal. Parigi, Francia.
} 
alternativo di accoglienza, la discussione, come prevedibile, si è rapidamente orientata, per prossimità geografica e tematica, verso la situazione di Calais.

Di fronte alle politiche di inospitalità e di marginalizzazione messe in atto dalle istituzioni a tutti i livelli, di fronte allo smantellamento della jungle e alla costruzione nel marzo scorso di un "campo" di accoglienza ${ }^{2}$ semi-chiuso, sottoposto a controlli estremamente rigidi (identificazione palmare ecc.) e in grado di accogliere circa 1500 persone, meno di 1/3 dei migranti presenti nell'area $^{3}$, mi sono permesso di evocare la possibilità, come evidenziato dall'antropologo Michel Agier in un suo intervento pubblico ${ }^{4}$, che la stessa jungle, l'accampamento auto-organizzato dai "migranti", che prima dello sgombero offriva riparo a almeno 4000 persone, rappresentasse una condizione di vita preferibile alla sua distruzione e alla costruzione del centro "Jules Ferry"

Preferibile perché, a dispetto del relativo isolamento e della ghettizzazione dell'agglomerato "extra" urbano ${ }^{6}$, sorto in uno spazio acquitrinoso periferico, che raccoglieva migranti di provenienze diverse (in particolare eritrei, sudanesi, afghani o ragazzi provenienti dall'Africa subsahariana), in condizioni amministrative diverse (sans papiers, richiedenti asilo o titolari di protezione) e con percorsi diversi (nuovi arrivati e migranti "in orbita" da anni, minori isolati, giovani adulti, qualche famiglia), la jungle era diventata un luogo di socialità, dove una comunità eterogenea, precaria e marginalizzata si ritrovava, si organizzava, e tentava di perennizzare un'installazione, di costruire uno spazio abitabile, vivibile, in un orizzonte di esclusione ${ }^{7}$.

Anche con la collaborazione, talvolta raffazzonata, talvolta ideologica, di attivisti e militanti ${ }^{8}$, e con il sostengo di una parte della cittadinanza ${ }^{9}$, i cosiddetti

2 Mi permetto di utilizzare questa formula intermedia per classificare la soluzione ibrida di un centro di semi-detenzione (tra il centro di accoglienza per richiedenti asilo e il centro di detenzione amministrativa, CRA in Francia) che in una situazione "emergenziale" e di "eccezionalità giuridica" prende progressivamente la dimensione e la forma dei campi profughi gestiti dalle organizzazioni militaro-umanitarie fuori dai confini europei (Agier). La recente idea della sindaca di Parigi Hidalgo di creare a Parigi un campo profughi reppresenta una delle cosneguenze estreme di questa logica.

3 RODIER, Claire. Migrants et refugiés. Réponse aux indécis, aux inquiets et aux réticents.

4 Cf. <http://www.lemonde.fr/idees/article/2016/02/14/ne-detruisons-pas-le-bidonville-de-calais 4865067_3232.html\#U9LZplc4y756A6MT.99>.

5 La situazione a Calais rimane in divenire, perché la comunità migrante, arroccandosi intorno ai pochi spazi risparmiati dalla demolizione, hanno ricominciato a ricostruire, a installarsi e organizzarsi di nuovo.

6 In senso urbanistico e politico, al di fuori del tessuto urbano e della cité (BALIBAR, Etienne. Droit de cité).

7 La questione degli insediamenti di migranti sul litorale di Calais e degli interventi istituzionali per impedirli, smantellarli o gestirli è annosa e risale quantomeno alla vicenda del campo di Sangatte (1999). Non è dato sapere ancora quanto il recente Brexit inciderà sulla sua evoluzione.

8 La deriva verso un turismo umanitario, a Calais come a Lesbos, è una constatazione oggettiva: il numero di associazioni, militanti, gruppi, collettivi etc. che intervengono "dall'esterno" spesso bypassando il ruolo degli attivisti locali - che quantomento conoscono la situazione sul territorio - si moltiplica e si concretizza, proponendo una variante minoritaria dell'ingerenza militare, economica e politica della ragione umanitaria istituzionale (Fassin, Duffield, Agier).

9 Cf. <www.passeursdhospitalites.wordpress.com>. 
"lieux de vie" avevano iniziato a prendere l'aspetto di abitazioni, con spazi comuni e altri in cui un minimo di intimità diventava possibile; erano sorti dei luoghi di culto, in una sorta di felice coabitazione empirica, e anche degli esercizi commerciali, delle forme di auto-organizzazione economica. I diversi gruppi erano riusciti a polarizzare lo spazio, a connotarlo e differenziarlo, sviluppando un embrione di società, una forma possibile di inurbamento ${ }^{10}$. Ovviamente, questa autogestione non era al riparo da tensioni e conflitti interni, e non poteva non replicare dinamiche presenti in altri contesti, altre situazioni: presenza di cosiddetti "passeurs", situazioni di violenza e sfruttamento, semplice tensione generata dalle condizioni di sopravvivenza deprecabili in cui erano costretti a far fronte.

Un rappresentante della "ragione umanitaria"11, operatore direttamente implicato in uno dei tanti insediamenti disseminati sul litorale nella gestione sul territorio dell' "emergenza" (perché il lessico risulta invariabile in Francia, in Italia, in Grecia o altrove) ha ritenuto opportuno intervenire per criticare questa preferenza, l'evocazione argomentata di questa possibilità, in nome di una presunta "estetizzazione" della presenza migrante, delle dinamiche in atto, del luogo stesso come spazio di sperimentazione: l'argomento, piuttosto banale, era quello che incensare una relativa autonomia e una forma non convenzionale di organizzazione (che si sottraeva alla logica gestionale imperante) significava ignorare e quasi avallare la presenza all'interno della jungle di disfunzionamenti, di violenze, di "insicurezza". Difunzionamenti, sempre secondo questa voce della ragione umanitaria, che una presenza organizzata di soggetti istituzionali (dell'umanitario e del militare) avrebbe provveduto se non a risolvere, quantomeno a gestire, o neutralizzare. In sostanza, la prospettiva dell'umanitario istituzionale sostiene il suo diritto/dovere di ingerenza e di intervento in situazioni come quelle della jungle di Calais, in nome della sicurezza, per i cittadini e per gli stessi migranti, attraverso forme più o meno violente di controllo e repressione, imponendo un modello di gestione che delegittima ogni forma di collaborazione o organizzazione alternativa, di gruppi militanti come i NoBorders o degli stessi migranti.

\section{Produzione di passività}

Soggiacente all'idea che l'ingerenza dell'umanitario (la sua governamentalità) sia funzionale (e strumentale) alla neutralizzazione di elementi di crisi (reale o potenziale) e all'organizzazione/gestione controllata di una realtà a partire da un modello "imposto a fin di bene"12 come nella migliore

\footnotetext{
${ }^{10}$ Cf. <https://www.theguardian.com/artanddesign/2016/jun/08/refugees-calais-jungle-camp-arc hite cture-festival-barbican $>$.

11 FASSIN, Didier. La Raison Humanitaire.

12 ZIZEK, Slavoj. Against Human Rights.
} 
delle tradizioni autoritarie, coloniali e neocoloniali, emerge qui in modo flagrante la concezione radicalmente passiva del migrante, punto di partenza di questa breve riflessione sull'idea di agire migrante. Perché per considerare i margini di azione, sempre più esigui e condizionati, di un'umanità migrante, è necessario interrogare preliminarmente l'impatto e la violenza di un sistema che sembra organizzato per neutralizzarli sistematicamente ${ }^{13}$.

Secondo un ragionamento paradossale, data la sua (spesso supposta e comunque generalmente indotta) non-autonomia esistenziale e pratica in situazione migratoria (abitativa, economica, sociale) e la sua presenza tollerabile solo quando reificata, oggettivata in vittima o in persona destinataria - dopo debita inquisizione - di una qualche forma di protezione giuridica e sussistenza, che degenera sovente nell'assistenziale, diventa pressoché automatica la totale delegittimazione di qualsivoglia forma di iniziativa che possa assumere i toni di un azione politica, autonoma, rivendicativa, "produttiva" da parte del migrante, a prescindere dalla sua iscrizione "topologica" in categorie di gestione ${ }^{14}$. Quella che potrebbe essere recepita, come nel caso di Calais, come azione politica rivendicativa e significativa, elaborata da una collettività produttiva, è ridotta al rango di deriva di ordine pubblico, di disfunzionamento organizzativo, di forma estetizzata, o di "eccezione" intollerabile in una logica di gestione umanitaria che non contempla l'espressione di una soggettività migrante ${ }^{15}$ : perché, funzionando specularmente al suo corrispettivo sicuritario - che agisce criminalizzando, clandestinizzando e invisibilizzando -, ha come ultimo obiettivo quello di produrre e gestire una umanità resa passiva e neutralizzata ${ }^{16}$.

In sostanza, la logica dominante è quella di una riduzione del migrante a soggetto di non-diritto ${ }^{17}$, che può affidarsi solo passivamente all'applicazione sul suo corpo di una protezione umanitaria che strumentalizza la retorica dei

${ }_{13}$ SAYAD, Abdelmalek. Immigration et "pensée d'État".

${ }^{14}$ NYERS, Peter. Rethinking Refugees. Beyond State of Emergency.

${ }^{15} \mathrm{Si}$ intenda per soggettività migrante non una dimensione collettiva stabile e omogenea, bensì la condizione umana in grado di costituirsi e rappresentarsi come soggetto agente in un orizzonte migratorio, dunque in movimento. Soggettività differenti di innumerevoli traiettorie individuali che convergendo e raggruppandosi lungo queste traiettorie possono organizzarsi ed assumere una portata collettiva, temporanea e legata alla variabilità delle stesse traiettorie, come nel caso di Ventimiglia o di Idomeni, o più strutturata, come nel caso delle frontiere di Ceuta e Melilla o di Calais.

16 Il funzionamento del dispositivo militare-umanitario che ha contraddistinto e contraddistingue gli interventi dei paesi occidentali nel mondo nel nome dell'esportazione di democrazia e sviluppo, con le conseguenze che conosciamo, è stato messo in risalto da numerosi autori (DUFFIELD, Mark. Global Governance and the New Wars: The Merging of Development and Security; ESCOBAR, Arturo. The Invention of Development); la sua declinazione nell'orizzonte europeo, tra "guerra ai migranti" e business dell'accoglienza (PALIDDA, Salvatore. Polizia post-moderna) esplicita ancora di più la funzione strumentale della retorica umanitaria come vettore di gestione e di controllo delle popolazioni, e mette in risalto ancora di più tutte le sue contraddizioni.

17 DAL LAGO, Alessandro. Non-persone. L'esclusione dei migranti in una società globale; PALIDDA, Salvatore. Délit d'immigration. 
diritti umani per affinare il suo dispositivo di captazione, la sua logica gestionale che assume sempre più i caratteri di una logistica dell'umanitario ${ }^{18}$.

Focalizzare I'attenzione sulla categoria dei rifugiati (o potenziali tali) e dunque accettare questa unilaterale grammatica dei diritti umani, relega tuttavia ancora ai margini dell'orizzonte sociale e politico quei migranti che, definiti "economici", risultano semplicemente un resto da respingere ${ }^{19}$, espellere, oppure integrare "invisibilmente" come forza-lavoro nero, in percorsi di clandestinizzazione e sfruttamento.

In entrambi i casi, appare evidente come il dispositivo di controllo e gestione della migrazione proceda attraverso una produzione progressiva di passività che garantisca una "presa" su questa umanità marginalizzata, che diventa o, come detto, mera forza-lavoro sfruttabile, o corpo/vittima da mantenere in vita per alimentare un'economia speculativa dell'umanitario e dell'accoglienza.

Se questa negazione del soggetto "di diritto" risulta flagrante e problematica, e giustifica un'attenzione particolare da parte di associazioni e società civile (Asgi, Naga, Gisti, HRW), è chiaro che una riflessione sulla soggettività migrante e le sue potenzialità operative, la sua agency ${ }^{20}$, individuale o collettiva, deve integrare la dimensione giuridica del diritto negato in uno spettro più ampio, che non sia quello della sola legalità, ma si estenda ad una rivendicazione di legittimità.

Il che vuol dire considerare non solo i limiti, le restrizioni e le condizioni ad una presenza "legale" sul territorio (il diritto di esserci), ma anche il funzionamento del dispositivo di desoggettivazione e di "depersonalizzazione" operativo e organizzato per inibire e neutralizzare quelle che potremmo considerare le "condizioni di agentività" della soggettività migrante (la possibilità e la modalità dell'esserci) ${ }^{21}$.

Parafrasando Spivak ${ }^{22}$, prima di entrare brevemente nel merito delle modalità di azione di questa soggettività, possiamo infatti chiederci: "can migrants act?", ovvero in quale orizzonte politico, sociale, culturale l'azione migrante risulta possibile, prima ancora che legittima o legale? Constatata la quasi totale non esistenza giuridica, se non residuale, della figura del migrante (non ci riferiamo qui all'“immigrato" già insediato sul territorio e relativamente integrato, "emerso" e visibile a livello amministrativo), non possiamo tuttavia limitarci ad imputare esclusivamente ad essa la difficoltà riscontrata dalla soggettività migrante a mettersi in atto, a tradursi in azione. Non aver "dritto a" non significa "non poter fare".

\footnotetext{
${ }^{18}$ LUSSAULT, Michel. De la lutte des classes à la lutte des places.

19 BAUMAN, Zygmunt. Wasted Lives: Modernity and Its Outcasts.

${ }^{20}$ GIDDENS, Anthony. New rules of sociological method; IDEM. The constitution of society: outline of the theory of structuration.

21 IVEKOVIC, Rada. Réfugié-E-s, les jetables.

${ }^{22}$ SPIVAK, Gayatri Chakravorty. Can the Subaltern Speak?
} 


\section{Atti di presenza}

Diventa interessante a questo proposito operare uno slittamento dal piano del diritto verso una dimensione meno normata e circoscritta, che potremmo definire esistenziale, per la quale scomodiamo la nozione di presenza/crisi di presenza elaborata dall'etnologo italiano Ernesto De Martino ${ }^{23}$ : se la "presenza" per De Martino consiste nella capacità di "essere al mondo", ovvero di affermare la propria esistenza attraverso un agire inscritto nel contesto sociale e culturale di riferimento, la crisi della presenza assume i connotati dell'incapacità di agire, di prendere parte e posizione nel presente, e di elaborare una progettualità rivolta verso il futuro. Benché De Martino non si riferisse ad un orizzonte transculturale di migrazione, appare evidente la possibilità di applicare questa nozione alla condizione migrante contemporanea.

Se il percorso migratorio, disseminato di ostacoli e di situazioni limite in cui il rischio di perire o di scomparire è quanto di più attuale, è caratterizzato quantomeno nelle sue fasi iniziali dalla possibilità di elaborare proiezioni rispetto a una "salvezza" o semplicemente a un futuro migliore, investendo la dimensione essenziale del desiderio come pulsione che spinge ad agire, il dispositivo/frontiera e i suoi corollari di gestione della migrazione (espulsione, esclusione, accoglienza, ...), che funziona come abbiamo evidenziato attraverso la produzione di "vittime" o di "indesiderabili", interviene non solo a sottomettere la figura del migrante a una logica giuridica dell'esclusione, ma procede a neutralizzare la soggettività migrante, inscrivendola in una gestione meccanica (Hotspot, rilocalizzazione, Dublino, ..) che nega ogni possibilità e la stessa capacità di elaborare e sviluppare una progettualità, una proiezione del desiderio, una immaginazione orientata verso il futuro.

Non è casuale che i migranti, obbligati a lasciare la jungle di Calais, siano stati in parte dirottati, spesso con false promesse o con ricatti, verso "centri di accoglienza" sparpagliati sul territorio francese che prendono il nome di CAO, centri di accoglienza e orientamento: ora, I'accampamento di Calais era connotato esattamente dalla volontà e dalla capacità da parte dei migranti di "orientarsi" autonomamente, in questo caso verso la Gran Bretagna, o comunque di posizionarsi in una situazione di transito possibile che testimoniava la loro determinazione e la loro capacità assumere una decisione, prendere una posizione e una direzione. $E$, più in generale, rappresentava la materializzazione della rivendicazione di una libertà di scegliere, e quindi di agire, che travalicava i limiti, le restrizioni e le condizioni imposte dalle istituzioni.

Estirparli dal luogo-orizzonte che rappresenta un punto simbolico fondamentale nelle loro auto-narrazioni e nelle loro proiezioni significa respingerli materialmente, ricacciarli indietro, ma soprattutto non riconoscere

${ }^{23}$ DE MARTINO, Ernesto. II mondo magico: prolegomeni a una storia del magismo; IDEM. Morte e pianto rituale nel mondo antico: dal lamento pagano al pianto di Maria. 
loro la capacità di desiderare e di operare in funzione di questo desiderio. Significa delegittimare questa energia vitale, ignorandone la portata politica, ma soprattutto aggredire e reprimere proprio questa capacità di elaborazione, questa cifra di presenza al mondo che viene sistematicamente neutralizzata, sterilizzata. In sostanza, la negazione sistematica di una libertà di agire (ridotta spesso al mero diritto di sopravvivere) passa per una violenta e altrettanto sistematica desoggettivizzazione della persona, per una neutralizzazione della sua identità, della sua biografia, delle sue competenze. Ridotto a una cifra in una gestione di flussi, a un dossier in una banca dati del sistema di identificazione, espulsione e distribuzione sul territorio, a materia vivente passiva da gestire, il migrante non è solo privato della libertà di agire nel presente, ma prima di tutto della facoltà di immaginare e desiderare, di proiettarsi e elaborare un futuro. La dimensione personale, intima, affettiva viene totalmente negata, la provenienza e la biografia diventano discriminanti esclusivamente per attribuire uno statuto di vittima o di indesiderabile, la parola e la memoria sono oggetto di inchiesta, di interrogatorio, di diffidenza. In questo orizzonte, che non contribuisce ad attutire eventuali traumi precedenti, ma li lascia macerare nel silenzio, e ne produce invece di nuovi, la stessa presenza, ben prima della capacità di agire, entra in crisi, e si innescano meccanismi di invisibilizzazione e auto-marginalizzazione, fonte di nuova sofferenza.

L'impatto disastroso di questo dispositivo di neutralizzazione attraverso la criminalizzazione, la marginalizzazione o la vittimizzazione, che si attacca tanto alle relazioni sociali disgregando le dimensioni collettive e molecolarizzando le esperienze, quanto alla dimensione individuale (familiare, relazionale), e che ha una ricaduta significativa sui margini di un agire migrante, emerge inesorabilmente dalla moltiplicazione sintomatica di situazioni di disagio psichico ed esistenziale estremo ${ }^{24}$ che una certa etnopsochiatria "istituzionale" fatica a riconoscere: perché presupporrebbe di mettere in discussione radicalmente dall'interno il sistema di dominazione e violenza dell'umanitario che con l'argomento di trattare e intervenire su traumatismi esogeni - il male altrove, il male precedete - evita accuratamente di prendere in considerazione e di problematizzare il traumatismo che esso stesso produce al suo interno (a proposito si vedano le posizioni critiche di MSF25).

La volontà istituzionale di sradicare con la violenza questa comunità migrante per disperderla, diminuendo in questo modo il suo impatto concreto,

\footnotetext{
${ }^{24}$ ACHOTEGUI, Joseba. Emigrar en situación extrema. El Síndrome del inmigrante con estrés crónico y múltiple (Síndrome de Ulises); BENEDUCE, Roberto. Un lugar en el mundo. Senderos de la migración entre violencia, memoria y deseo; IDEM. Undocumented bodies, burned identities: refugees, sans papiers, harraga when things fall apart.

25 Si rimanda alla recente pubblicazione da parte di MSF Italia del rapporto Traumi ignorati (cf. <http://archivio.medicisenzafrontiere.it/pdf/Rapp_Traumi_Ignorati_140716B.pdf >).
} 
assume toni grotteschi quando pretende di imporle vettori di movimento e di presenza forzati, di orientare in modo coercitivo secondo una volontà e una progettualità estranea ai soggetti interessati, che sono percepiti e gestiti come entità passive sprovviste - private - di volontà e di prospettive indipendenti, siano esse individuali o collettive.

La gestione emergenziale dell'accoglienza in Italia a partire dal 2011 (emergenza Nordafrica) che ha generato la proliferazione dei Centri di accoglienza straordinari (CAS) risponde a questa logica di una neutralizzazione per disseminazione: obbligati a risiedere in strutture isolate, lontano dai centri urbani e da condizioni normali di socializzazione, i migranti dopo qualche giorno cercano di abbandonare le strutture e continuare la loro traiettoria, spesso a prescindere dall'evoluzione del loro dossier amministrativo (alterego virtuale inscritto nel sistema di gestione). E le argomentazioni volte a spiegare questi comportamenti di "resistenza" chiamano in causa spesso la "veridicità" delle domande d'asilo e della condizione di profugo invece che interrogare la struttura dell'accoglienza e questa negazione di autonomia decisionale: come se chiedere aiuto implicasse di rinunciare a ogni pretesa, a ogni progettualità, ad una autodeterminazione.

\section{Agire sovversivo}

Di fronte a questo dispositivo di neutralizzazione, l'agency migrante assume in questo orizzonte toni che non sono più quelli di una capacità appunto di autodeterminazione indipendente, positiva e "comprensibile", inscrivibile in una grammatica condivisa con l'ambiente circostante ${ }^{26}$, ma quelli, in una prospettiva più foucaultiana, di una elaborazione strategica di forme e di modalità di autonomia in un contesto di "assoggettamento".

Detto altrimenti, in una situazione di esclusione, marginalizzazione e passività diventa più difficile articolare la propria posizione liminare rispetto a quelle di altri segmenti della società e conformemente alle condizioni di confronto date, per il semplice fatto che la stessa società (le sue istituzioni come la popolazione) non attribuisce e non garantisce a questi elementi "esteriori" la possibilità di inter-agire, di rivendicare e di negoziare apertamente la propria posizione e il proprio ruolo. In un panorama di ripiegamento identitario e di irrigidimento xenofobo nei confronti dell'alterità, non si tratterà quindi di organizzare forme di resistenza (che presupporrebbe una presenza messa in discussione e minacciata di esclusione) ma piuttosto di alimentare e sperimentare strategie e modalità di "esistenza" collaterale, di auto-organizzazione e di autosufficienza in una dimensione paradossale di presenza assente, di esclusione inclusiva, per utilizzare la definizione dell'eccezione data da Agamben ${ }^{27}$.

\footnotetext{
${ }^{26}$ BOURDIEU, Pierre. Outline of a theory of practice.

${ }^{27}$ Forme che oscillano tra l'invisibilità impercettibile e l'emersione conflittuale, tra la l'illusione
} 
Si tratta di modalità che talvolta non corrispondono per nulla a quelle codificate e coerenti previste o prevedibili dal sistema, e che la stessa società civile tende a sollecitare, stimolare, sostenere: il tentativo da parte dei sindacati in Italia e in Francia di pilotare e assorbire le rivendicazioni dei sans-papiers inscrivendole in una grammatica politicamente connotata, presenta in questo senso delle criticità importanti; perché senza entrare nel merito dei diversi gradi e modi di politicizzazione delle diverse comunità migranti, questa presenza e questa potenzialità non dovrebbe essere tradotta per divenire strumentalizzabile, come troppo spesso avviene, ma recepita inserita in una dinamica di condivisione strategica di progettualità. Gli slanci esterni ("interni" al nostro orizzonte politico sociale) volti a organizzare e disciplinare l'agire migrante, inscrivendolo in un alveo di lotte e rivendicazioni codificate, peccano spesso di una ingenuità "etnocentrica" che non è in grado di percepire le potenzialità e le caratteristiche di altre modalità operative, di altre energie, di altre forme di organizzazione.

Altre volte le modalità di azione risultano semplicemente invisibili, non perché siano segretate (e dunque evidentemente sospette agli occhi dei sistema), ma perché risultano impercettibili e incomprensibili per uno sguardo istituzionale: anni di percorsi migratori verso l'Europa, dall'Africa occidentale verso la Spagna, nel Mediterraneo centrale, verso oriente tra Turchia, Grecia e rotta balcanica, hanno prodotto, oltre a innumerevoli tragedie e un numero intollerabile di vittime, anche una proliferazione e condivisione di un "sapere" migrante che supporta una agency conseguente.

È necessario sottolinearlo per sfatare il mito che tutta la dinamica migratoria sia gestita da passeurs e smugglers (altro modo per negare una prospettiva migrante soggettiva in grado di costruire progetti, agire, improvvisare, modificare, scegliere), mentre fare appello o affidarsi a tali figure (prodotte, anch'esse come tali dalla narrazione sicuritaria) è semplicemente una fase, un'operazione tecnica, spesso molto ben articolata anche da parte del migrante, all'interno di una strategia più vasta interpretata singolarmente (o in un progetto familiare $\mathrm{o}$ comunitario condiviso).

Tra le comunità afghane installate tra Patrasso e Igoumenitsa prima dell'apertura della rotta balcanica, una efficace trasmissione di informazioni e "istruzioni" faceva parte della "staffetta" organizzata per accogliere i nuovi arrivati, inscriverli in una dinamica collettiva di sostentamento e agevolare i più anziani nelle operazioni di passaggio.

Un'organizzazione analoga nelle città di transito in Marocco come Fès e alle porte delle enclavi spagnole di Ceuta e Melilla, permette ai "vecchi" migranti in attesa di attraversare la frontiera di contare sui servizi "offerti" dai nuovi venuti.

dell'assenza e la materializzazione delle presenza. Cf. AGAMBEN, Giorgio. Homo Sacer. Il potere sovrano e la nuda vita. 
I nuovi arrivati prendono in carica la gestione e l'approvvigionamento delle risorse, la risoluzione dei problemi materiali di questa comunità fluida (che esiste sempre diversa e sempre uguale) per permettere a chi è arrivato prima di loro di dedicare le proprie energie esclusivamente al passaggio. Si tratta di una "passazione" che non è priva di tensioni, in cui si concretizzano forme di prevaricazione e di violenza, ma che denota una forma di organizzazione "in frontiera" e "in movimento" e che delinea forme di solidarietà e collaborazione possibile al di fuori della grammatica dell'umanitario.

Queste forme di cooperazione si dipanano e si configurano e riconfigurano in base alle evoluzioni delle traiettorie, attraverso connessioni virtuali e telematiche ${ }^{28} \mathrm{o}$ attraverso la trasmissione di pratiche e tecniche puntuali, o I'organizzazione di reti di solidarietà e di sostegno tappa dopo tappa, in una dimensione di concretezza che relativizza la distinzione tra legalità e illegalità e che si fonda appunto sull'adeguamento continuo, sull'aggiornamento di un'agency "in movimento" che punta meno sull'interazione e sulla ripetizione che sulla differenziazione e l'adattabilità.

Collateralmente constatiamo come il violento attacco istituzionale a numerose realtà della società civile (in Italia come in Francia o altrove) che si smarcano dalle posizioni assistenziali e paternalistiche e si attivano a sostegno dei migranti (nelle loro diverse situazioni e nei diversi punti focali delle loro traiettorie) rispetto alle loro rivendicazioni e alle loro progettualità, resistendo a una dinamica di "transfert" della buona coscienza, dimostra paradossalmente la validità di questo approccio che rifiuta tanto la dimensione gestionale dell'umanitario quanto un sostegno per assorbimento, per riconoscere una autonomia decisionale all'agire migrante ${ }^{29}$ : approccio che risulta dunque sedizioso in quanto solidale con una soggettività migrante non codificata ${ }^{30}$.

\section{Spazi e linee di azione}

Schematizzando molto e riferendoci ad un orizzonte che interroga la dimensione soggettiva, in termini di desiderio, di capacità di proiezione e di azione $^{31}$, potremmo inserire questo agire migrante, che quindi ha una matrice di alterità e di "differenza" attiva da tenere costantemente presente, in una tensione tra un piano di desoggettivazione, assoggettamento e neutralizzazione, tendente a ridurre il migrante a ente passivo, che non può e non è in grado di

\footnotetext{
${ }^{28}$ DIMINESCU, Dana. The Connected Migrant: An Epistemological Manifesto.

${ }^{29}$ Si veda l'approccio articolato nella Carta di Lampedusa (2014) o della guida Welocme to Italy (2015).

${ }^{30}$ La criminalizzazione dei NoBorders di Calais, contro i quali si è montato un circo politico mediatico di diffamazione, di accuse e di ritorsioni, ha echi un po' dovunque in Europa: alla frontiera greco-macedone come a Ventimiglia, nel nord est Italiano come in Spagna.

${ }^{31}$ FOUCAULT, Michel. Histoire de la sexualité. 1, La volonté de savoir; BUTLER, Judith, LACLAU, Ernesto, ZIZEK, Slavoj. Contingency, Hegemony, Universality: Contemporary Dialogues on the Left.
} 
agire, (perché vittima o indesiderabile) ed un piano di operatività "mediato", che passa per forme più o meno esplicite di "integrazione" o sussunzione in dinamiche, comportamenti, pratiche della società di accoglienza: piano in cui l'agire è tradotto e comprensibile, accettabile e inscrivibile in un modello generale e dunque formalmente limitato, controllato, inglobato e gestito.

Questo spettro estremamente vasto include modalità di reazione e di rifiuto radicali, sociali o patologiche, dinamiche di resilienza e di sopravvivenza, e dal versante opposto forme più o meno convenienti, tollerabili e gestibili di adattamento e di assimilazione, che includono adesioni volontarie ed entusiastiche, e risoggettivazioni traumatiche e problematiche in termini di coerenza culturale, trasmissione, evoluzione. Certo, nella misura in cui il processo migratorio è fragilizzato, la legittimità di presenza è messa in discussione, la crisi è latente o sempre imminente, la portata dell'agire, il suo impatto sull'evoluzione del quotidiano diventano meno significativi, o assumono contorni diversi.

La "finzione" e l'auto-rappresentazione secondo i canoni prescritti possono diventare parte integrante di questa postura "sospesa", e allora l'agire può risolversi in una pratica mimetica che asseconda la proiezione della società d'accoglienza. In questo senso le accuse verso i "falsi richiedenti asilo"32 convergono paradossalmente con le ingiunzioni del sistema a "farsi vittima", ad accettare e introiettare questa dimensione esistenziale che sola sembra concedere un diritto di presenza, o almeno di permanenza.

Era in un certo senso prevedibile che l'ipocrisia della ragione umanitaria e la sua dimensione speculativa entrassero in cortocircuito come sistema, per il fatto stesso di alimentare una doppia logica sempre discriminatoria di esclusione o di integrazione differenziale ${ }^{33}$, e che debbano di conseguenza essere riconsiderati e messi in discussione a livello politico e sociale alle fondamenta, con una necessaria revisione ad esempio delle nozioni di asilo e di rifugiato per come sono teorizzate nella Convenzione di Ginevra. Ma è evidente che questa tensione, questo orizzonte contraddittorio, ha marcato un vuoto all'interno del quale la presenza migrante è sottoposta ad una pressione costante e perenne, a un'instabilità (tra questi due poli di inclusione sempre condizionale e di esclusione sempre possibile) che, se in teoria potrebbe concedere spazi di azione e di invenzione, di fatto impatta sul quotidiano della migrazione in maniera violenta, destabilizzante e difficile da risolvere: come se essere migrante significasse essere in una condizione precaria, sempre sospesa, mai risolvibile.

Perché risulta estremamente difficile posizionarsi autonomamente e produttivamente, soggettivamente, in un piano inclinato che ha ad un

\footnotetext{
32 FASSIN, Didier. The Precarious Truth of Asylum; ROUSSEAU, Cécile, FOXEN, Patricia. Le mythe du réfugié menteur: un mensonge indispensable?

${ }^{33}$ MEZZADRA, Sandro, NEILSON, Brett. Border as Method, or, the Multiplication of Labor.
} 
estremo un farsi "vittima" o comunque essere accondiscendente alle categorie del sistema, in una sorta di habitus mimetico, funzionale e condizionante; e dall'altro forme di pura resistenza e chiusura che sfociano in situazioni di marginalizzazione ed esclusione estreme e irrevocabili.

In un contesto di discriminazione, la possibilità e la capacità di stare al gioco, "play the game", cioè di decifrare una situazione e strutturare un'azione conseguente che contempli il proprio desiderio, la propria volontà all'interno di un range di possibilità, non è scontata né evidente ed è inoltre sottoposta alla necessità di aggiornarsi in continuazione in risposta alle evoluzioni sistemiche ${ }^{34}$.

In questo orizzonte diventano degne di nota dunque delle modalità di azione minori ma che riescono a sovvertire la logica dominante e che traducono una versatilità ed un'adattabilità migrante al di fuori dagli schemi che gli si attribuisce, o che si intende imporgli: quando dei giovani assorbiti dal dispositivo umanitario come richiedenti asilo rivendono in un mercato rionale di Torino il loro kit di prima accoglienza per racimolare quattro soldi per proseguire il loro percorso, assistiamo ad un gesto che, al di là della facile indignazione degli attori dell'umanitario stesso, ha una portata simbolica che da una parte rivendica la volontà di non lasciarsi ingabbiare in categorie e percorsi predeterminati, e dall'altra irride il funzionamento del dispositivo stesso, che specula sull'attribuzione di valore all'accoglienza: come i famosi 35 euro al giorno a persona che la disinformazione generale suppone erogati direttamente ai migranti e che invece vengono intascati dai gestori dei centri di accoglienza in cambio di servizi irrisori.

Si tratta di pratiche che si attualizzano in continuazione e denotano una capacità di lettura e di adattamento estremamente efficaci: quando a fine 2015 si stava affermando in Europa il cosiddetto approccio hotspot, I'organizzazione di un sistema progressivo di selezione e smistamento dei migranti secondo categorie e nazionalità determinate (criterio in contraddizione con i principi internazionali dell'asilo $)^{35}$ e si imbastiva la logica fallimentare della relocaltion in Europa, con una recrudenscenza delle violenze e dei soprusi nelle strutture di identificazione (uso della forza per l'ottenimento delle impronte digitali), l'esplosione del business dell'accoglienza in Italia e il caos nelle isole hotspot greche come Lesbos, si sono articolate strategie operative che consentivano ai migranti di preseverare nel loro progetto migratorio senza essere fagocitati da questo dispositivo depersonalizzante. Alla fronteria italo-austriaca (Brennero o Tarvisio) si incrociavano persone che cercavano di spostarsi verso nord (quei "transitanti" che nel 2014 hanno attraversato I'Italia senza lasciare tracce anche con la connivenza delle istituzioni passando da Roma a Milano) ed altri, specialmente afghani e pakistani, che sia

\footnotetext{
${ }^{34}$ GOFFMAN, Erving. Strategic Interaction.

${ }^{35} \mathrm{Si}$ vedano in proposito i rapporti e le note di associazioni come Migreurop, La Cimade in Francia o Lasciatecientrare in Italia.
} 
per la progressiva chiusura della rotta balcanica che rimaneva percorribile solo per determinati gruppi (i siriani nello specifico), sia per una stima statistica che attestava per loro dei tassi di riconoscimento di protezione internazionale più elevati in Italia che in Germania in quel periodo, deviavano verso sud e dopo aver attraversato i Balcani si dirigevano verso Trieste o Gorizia, spesso sostando nei pressi della stazione di Udine, per presentare una domanda d'asilo.

Altre volte, come nel caso della jungle di Calais, si tratta di pratiche che "diventano" sovversive e dunque inaccettabili per il sistema, nella misura in cui mettono in atto e in mostra processi di coesione e collaborazione che interpellano i fondamenti politici dei paesi "ospitanti" (fondare una comunità), rimanendo purtanto esclusi dall'orizzonte della legittima presenza (evidenziando in questo modo le flagranti contraddizioni della società nella quale si inscrivono). L'ostinazione con cui la jungle di Calais si ricostituisce e si riorganizza dopo ogni sgombero e ogni smantellamento evidenzia una volontà che va al di là delle realistiche condizioni di praticabilità e delle restrizioni politiche, amministrative, sociali, e che spinge senza tregua verso un attestato di esistenza guadagnato sul campo.

Costruire una comunità e abitare uno spazio sottraendosi alle ingiunzioni istituzionali e resistendo alle violenze repressive significa assumere e affermare il proprio agire in una prospettiva in un contesto che va al di là e esula dalle norme vigenti, che crea un conflitto produttivo alla frontiera tra inclusione e esclusione $^{36}$ in grado di intervenire a modificare una situazione, di estendere ora non i limiti della cittadinanza, paradigma storico internamente in crisi, ma le condizioni di presenza e di residenza su un territorio. Significa non sottostare a una logica di inscrizione in categorie passive o di esclusione tout court, ma affermare la possibilità di articolare una "agency migrante", un agire storico che si inscrive in dinamiche di mobilità internazionale e di equilibri geopolitici ben più vasti delle logiche territoriali ed identitarie contemporanee.

Analizzare e riflettere sulle traiettorie migranti, sui percorsi e le tappe di una mobilità umana che rimette in discussione i paradigmi della mondializzazione ${ }^{37}$ e le dinamiche "classiche", relativamente statiche, di e/immigrazione ${ }^{38}$, significa dunque riflettere sulla variazione progressiva della capacità e della possibilità di una soggettività migrante di agire, ovvero di affermare e rivendicare una presenza attiva all'interno del contesto sociale in cui si trova, è costretto a rimanere (tra resistenza e resilienza) o si transita; significa ragionare in termini di versatilità, adattabilità e capacità di elaborare e aggiornare un progetto in divenire, un percorso non necessariamente lineare che procede per tappe e

\footnotetext{
${ }_{36}$ ISIN, Engin F. Being Political: Genealogies of Citizenship.

37 PALIDDA, Salvatore. Mobilità umane. Introduzione alla sociologia delle migrazioni.

${ }^{38}$ SAYAD, Abdelmalek. La double absence. Des illusions de l'émigré aux souffrances de l'immigré.
} 
tentativi, tenendo presente la complessa articolazione della concatenazione di situazioni locali in un orizzonte/traiettoria; significa distinguere tra produzione di invisibilità o al contrario esibizioni strumentalizzate dal sistema o da altri attori in campo, e strategie di presenza, di agire minoritario, di "divenire invisibile", o al contrario di sovraesposizione, di esibizione e di rivendicazioni di presenza sviluppate e integrate dalle soggettività migranti; significa infine considerare una dinamica che delinea forme nuove di organizzazione, in cui soggettività e collettività si intersecano in un orizzonte in cui gli stessi parametri della presenza e dell'azione non possono ridursi alle condizioni locali, ma vanno inscritte in una geografia più ampia di approccio alla spazialità, alla libertà di movimento o alla libertà, come nel caso di Calais, di restare dove ci si trova e di operare attivamente per rendere questa presenza umanamente prima ancora che giuridicamente degna e legittima.

\section{Bibliografia}

ACHOTEGUI, Joseba. Emigrar en situación extrema. El Síndrome del inmigrante con estrés crónico y múltiple (Síndrome de Ulises). Revista Norte de salud mental de la Sociedad Española de Neuropsiquiatría, v. V, n. 21. 2004, p. 39-53.

AGAMBEN, Giorgio. Homo Sacer. Il potere sovrano e la nuda vita. Torino: Einaudi, 1995.

AGIER, Michel; LECADET, Clara (a cura di). Un monde de camps. Paris: La Découverte, 2014.

AGIER, Michel. Gérer les indésirables. Des camps de réfugiés au gouvernement humanitaire. Paris: Flammarion, 2008.

AGIER, Michel. Humanity as an Identity and Its Political Effects (A Note on Camps and Humanitarian Government). Humanity: An International Journal of Human Rights, Humanitarianism, and Development, v. 1, n. 1, Fall 2010, p. 29-45.

BALIBAR, Etienne. Droit de cité. Paris: PUF, 1998.

BAUMAN, Zygmunt. Wasted Lives: Modernity and Its Outcasts. Cambridge: Polity, 2004.

BENEDUCE, Roberto. Un lugar en el mundo. Senderos de la migración entre violencia, memoria y deseo. Ciudad de Mexico: Universidad Autónoma de Tlaxcala - Escuela Nacional de Antropología e Historia (INAH-ENAH), 2015.

BENEDUCE, Roberto. Undocumented bodies, burned identities: refugees, sans papiers, harraga when things fall apart. Social Science Information, v. 47, n. 5, 2008.

BOURDIEU, Pierre. Outline of a theory of practice. London: Cambridge University Press, 1977.

BROSSAT, Alain. Résistance(s) et pouvoir(s) chez Michel Foucault. In CHEMLA, Patrick. Résistances et transferts. Toulouse: érès, 2004, p. 115-119.

BUTLER, Judith; LACLAU, Ernesto; ZIZEK, Slavoj. Contingency, Hegemony, Universality: Contemporary Dialogues on the Left. London/New York: Verso, 2000.

BUTLER, Judith; ATHANASIOU, Athena. Dispossession: the performative in the political. Cambridge, UK Malden, Massachusetts: Polity Press, 2013. 
CARNEMOLLA, Davide; FURRI, Filippo. Calais il limbo d'Europa. 29.07.2015. Disponibile su: <https://tutmonda.wordpress.com/2015/07/29/il-limbodeuropa-le-vite-intrappolate-e-umiliate-dei-migranti-a-calais/ $>$.

DAL LAGO, Alessandro. Non-persone. L'esclusione dei migranti in una società globale. Milano: Feltrinelli, 2004.

DE MARTINO, Ernesto. Il mondo magico: prolegomeni a una storia del magismo. Torino: Einaudi, 1948.

DE MARTINO, Ernesto. Morte e pianto rituale nel mondo antico: dal lamento pagano al pianto di Maria. Torino: Einaudi, 1958.

DIMINESCU, Dana. The Connected Migrant: An Epistemological Manifesto. Social Science Information, v. 47, n. 4, 2008, p. 565-579.

DUFFIELD, Mark. Global Governance and the New Wars: The Merging of Development and Security. London: Zed Books, 2001.

ESCOBAR, Arturo. The Invention of Development. Current History, v. 98, n. 631, 1999, p. 382-396.

FASSIN, Didier. The Precarious Truth of Asylum. Public Culture, v. 25, n. 1, 2013, p. 39-63.

FASSIN, Didier. La Raison Humanitaire. Paris: Hautes Etudes-Gallimard-Seuil, 2010.

FOUCAULT, Michel. Histoire de la sexualité. 1, La volonté de savoir. Paris: Gallimard, 1976.

GATTA, Gianluca. Corpi di frontiera. Etnografia del trattamento dei migranti al loro arrivo a Lampedusa, in Presenze internazionali. Prospettive etnografiche sulla dimensione fisico-politica delle migrazioni in Italia. AM, n. 33-34, 2012, p. 129-162.

GIDDENS, Anthony. New rules of sociological method. London: Hutchinson, 1976.

GIDDENS, Anthony. The constitution of society: outline of the theory of structuration. Great Britain: University of California Press, 1984.

GOFFMAN, Erving. Strategic Interaction. Philadelphia: University of Pennsylvania Press, 1969.

HANAPPE, Cyrille. À Calais un autre devenir ville. Multitudes, v. 61, n. 4, 2015, p. 15-17.

ISIN, Engin F. Being Political: Genealogies of Citizenship. Minneapolis: University of Minnesota Press, 2002.

IVEKOVIC, Rada. Réfugié - E-s, les jetables. Paris: Al Dante, 2016.

KÖNGETER, Stefan; SMITH, Wendhy (eds.). Transnational Agency and Migration Actors, Movements, and Social Support. New York, London: Routledge, 2015.

LAVIE, Smadar; NARAYAN, Kirin; ROSALDO, Renato. Creativity/Anthropology. Ithaca and London: Cornell University Press, 1993.

LUSSAULT, Michel. De la lutte des classes à la lutte des places. Paris: Grasset (Mondes vécus), 2009.

MEZZADRA, Sandro; NEILSON, Brett. Border as Method, or, the Multiplication of Labor. Durham and London: Duke University Press, 2013.

NYERS, Peter. Rethinking Refugees. Beyond State of Emergency. New York, USA, London, UK: Routledge, 2006. 
PALIDDA, Salvatore. Délit d'immigration. COST A2 Migrations, Bruxelles: Commission européenne, 1997.

PALIDDA, Salvatore. Mobilità umane. Introduzione alla sociologia delle migrazioni. Milano: Edizioni Cortina, 2008.

PALIDDA, Salvatore. Polizia post-moderna. Milano: Feltrinelli, 2000.

RODIER, Claire. Migrants et refugiés. Réponse aux indécis, aux inquiets et aux réticents. Paris: La decouverte, 2016.

ROUSSEAU, Cécile; FOXEN, Patricia. Le mythe du réfugié menteur: un mensonge indispensable? Evol psychiatr, v. 71, 2006, p. 505-520.

RYGLEL, Kim. Bordering solidarities: migrant activism and the politics of movement and camps at Calais. Citizenship Studies, v. 15, n. 1, 2011, p. 1-19.

RYGIEL, Kim. Politicizing camps: forging transgressive citizenships in and through transit. Citizenship Studies, Special Issue: Citizenship After Orientalism: An Unfinished Project, v. 16, n. 5-6, 2012, p. 807-825.

SAYAD, Abdelmalek. Immigration et «pensée d'État». Actes de la recherche en sciences sociales, v. 129, septembre 1999, p. 5-14.

SAYAD, Abdelmalek. La double absence. Des illusions de l'émigré aux souffrances de I'immigré. Paris: Seuil, 1999.

SAYAD, Abdelmalek. The suffering of the immigrant. Cambridge: Polity press, 2004.

SIGONA, Nando. Campzenship: reimagining the camp as a social and political space. Citizenship Studies, v. 19, n. 1, 2015, p. 1-15.

SPIVAK, Gayatri Chakravorty. Can the Subaltern Speak? In NELSON, Cary; GROSSBERG, Lawrence (eds.). Marxism and the Interpretation of Culture. London: Macmillan, 1988.

ZIZEK, Slavoj. Against Human Rights. New Left Review, n. 34, july-august 2005, p. 115-131.

Articolo ricevuto il 25.06.2016 Accettato per la pubblicazione il 10.08.2016

Received for publication in June $25^{\text {th }}, 2016$ Accepted for publication in August 10 $0^{\text {th }}, 2016$

ISSN impresso 1980-8585

ISSN eletrônico 2237-9843

http://dx.doi.org/10.1590/1980-85852503880004702 